\title{
Diagnóstico y tratamiento de las estenosis subglóticas en pediatría: experiencia en un hospital de alta complejidad
}

\author{
Diagnosis and treatment of pediatric subglottic stenosis: \\ experience in a tertiary care center
}

\author{
Dr. Hugo Alberto Botto y Dra. Cinthia Giselle Pérez \\ Colaboradores: Dr. Alejandro Cocciaglia ${ }^{a}$, Dra. Mary Nieto ${ }^{a} y$ \\ Dr. Hugo Aníbal Rodríguez ${ }^{a}$
}

\begin{abstract}
RESUMEN
La estenosis subglótica es una de las causas más frecuentes de obstrucción de la vía aérea en pediatría. El 190\% son secundarias a la intubación endotraqueal. El diagnóstico se sustenta en la clínica del paciente, la evaluación radiológica, la laringoscopía flexible y la endoscopía rígida de la vía aérea bajo anestesia general. Debe sospecharse en niños con dificultad respiratoria posextubación. La conducta terapéutica dependerá de la gravedad de la estenosis subglótica y de la sintomatología del paciente. Describimos nuestra experiencia en cuanto a las etiologías de las estenosis subglóticas, el diagnóstico, el tratamiento y la evolución de pacientes con esta patología.

Palabras clave: laringoestenosis, estenosis subglótica adquirida, estenosis subglótica congénita, fisura laringotraqueal, intubación intratraqueal.
\end{abstract}

\section{ABSTRACT}

Subglottic stenosis is among the most common causes of airway obstruction in children, $90 \%$ of which resulting from endotracheal intubation. The diagnosis is based on the patient's clinical, radiologic evaluation, flexible laryngoscopy and rigid airway endoscopy under general anesthesia. It must be suspected in children with respiratory distress after extubation. The therapeutic approach depends on the severity of the subglottic stenosis and the patient's symptoms.

rvicio de

Endoscopía

Respiratoria

del Hospital de

Pediatría "Prof. Dr.

Juan P. Garrahan".

Correspondencia:

Dra. Cinthia

Giselle Pérez:

cinthiagperezent@gmail. com

\section{Financiamiento:}

Ninguno.

Conflicto de intereses:

Ninguno que declarar.

Recibido: 16-9-2014

Aceptado: 19-1-2015 traqueostomía en niños menores de 1 año. ${ }^{1}$

La subglotis se extiende por debajo de las cuerdas vocales hasta el margen inferior del cartílago cricoideo. ${ }^{1} \mathrm{Un}$ diámetro subglótico menor de $4 \mathrm{~mm}$ en un recién nacido se considera ES. ${ }^{2}$

Las causas de las ES pueden ser congénitas o adquiridas. Es congénita cuando no existe el antecedente de intubación. ${ }^{1}$ La ES congénita es la tercera anormalidad congénita laríngea más frecuente, luego de la laringomalacia y la parálisis cordal. El diagnóstico de ES adquirida se realiza cuando existe un antecedente de intubación, que es la forma más común $(90 \%) .{ }^{1} \mathrm{La}$ incidencia de estenosis laríngea posintubación endotraqueal es de $0,9 \%$ a $8,3 \%$, según distintos autores. ${ }^{1,2}$ Es más frecuente en la infancia, ya que la subglotis es la zona más estrecha de la vía aérea pediátrica. Últimamente, esta patología se encuentra en aumento como resultado del mejoramiento en la supervivencia de los pacientes asistidos en las unidades de cuidados intensivos. $^{2}$

El diagnóstico de ES se sustenta en la clínica del paciente, la evaluación radiológica, la laringoscopía flexible y la endoscopía rígida de la vía aérea bajo anestesia general.

Lo habitual es encontrar un paciente con cuadro obstructivo de la vía aérea alta (estridor, disnea y tiraje supraesternal y subcostal). Puede acompañarse de disfonía, afonía o llanto débil. Se debe sospechar en 
niños con fallos en la extubación o con necesidad de reintubación. ${ }^{1}$

La radiografía de cuello de perfil permite evaluar el calibre de la vía aérea y pone de manifiesto la presencia y longitud de las ES (Figura 1). La tomografía computada y la resonancia magnética nuclear proporcionan información del sitio y la longitud de la estenosis, pero no son solicitadas de forma rutinaria. ${ }^{2}$

La laringoscopía flexible valora anormalidades supraglóticas y la movilidad cordal. ${ }^{2}$ No es de gran utilidad para valorar la región subglótica.

El estándar de oro para el diagnóstico de ES es la endoscopía rígida bajo anestesia general, con laringoscopios de comisura anterior (Holinger) para exponer adecuadamente la subglotis. ${ }^{2} \mathrm{Se}$ evaluará la localización anatómica de la estenosis laríngea, el porcentaje de obstrucción subglótica, la longitud y consistencia (bajo palpación) de la ES.

La conducta terapéutica estará determinada por la gravedad, localización y consistencia de la ES y puede ser conducta expectante, dilataciones laríngeas, traqueostomía o cirugías laríngeas a cielo abierto. ${ }^{2}$

Figura 1. Radiografía de cuello de perfil; se observa, a nivel subglótico, un estrechamiento de su luz

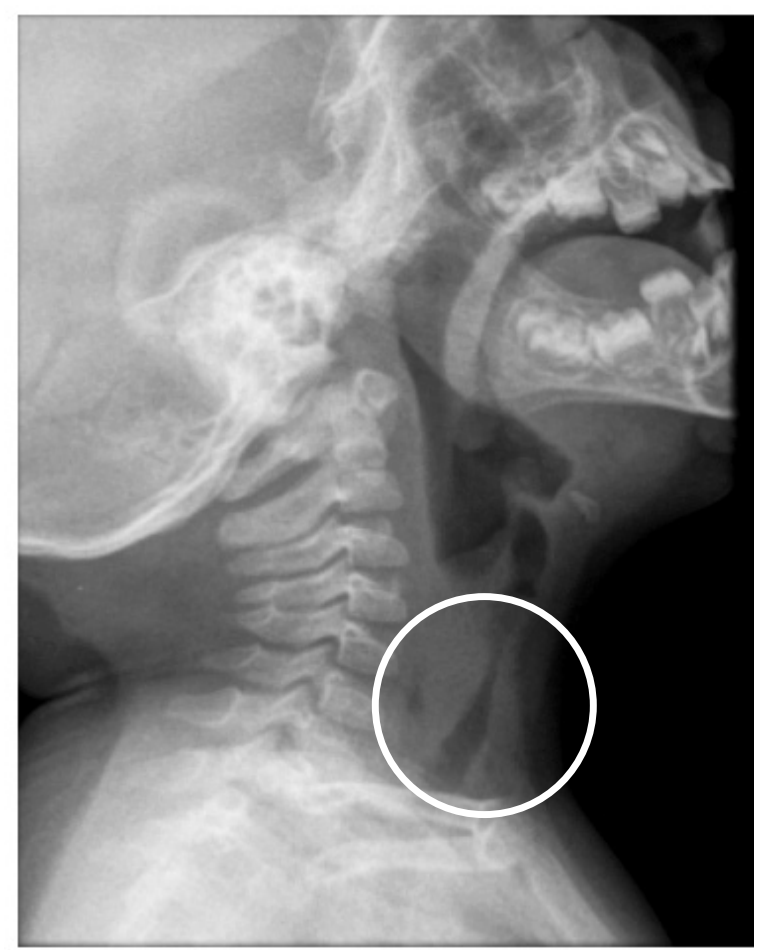

El objetivo del estudio es describir nuestra experiencia en cuanto a las etiologías de las ES, el diagnóstico, el tratamiento y la evolución de pacientes con esta patología.

\section{MATERIALES Y MÉTODOS}

Se reclutaron pacientes con diagnóstico de estenosis subglótica a quienes se les hubiera realizado una valoración de la vía aérea bajo anestesia general y/o algún procedimiento o cirugía laríngea abierta en el Servicio de Endoscopía Respiratoria del Hospital de Pediatría "Prof. Dr. Juan P. Garrahan" entre junio de 2012 y diciembre de 2013. Se diseñó un estudio descriptivo-retrospectivo. Se incluyeron aquellos pacientes tratados de manera expectante o de forma quirúrgica, ya sea con dilataciones laríngeas u operaciones laríngeas a cielo abierto.

Según lo recolectado de las historias clínicas electrónicas, se confeccionó una planilla MS Office Excel2007 ${ }^{\circledR}$ para su posterior análisis. El estudio fue aprobado por el Comité Revisor del Departamento de Docencia e Investigación de la Institución.

Todos los pacientes candidatos a cirugía laríngea abierta fueron valorados bajo anestesia general para determinar movilidad cordal (con el paciente en respiración espontánea), grado, tipo, localización y extensión de la estenosis. Luego de esta valoración, se decidió el tipo de cirugía por realizar.

Se utilizó la clasificación de Cotton y Myer, en la que una estenosis grado I tiene un compromiso menor del 50\% de la luz subglótica; grado II, del 51-70\%; grado III, del 71-99\%; y grado IV, del $100 \%$ (o atresia laríngea) ${ }^{2}$ (Figura 2).

Las dilataciones laríngeas incluyen la utilización de dilatadores ureterales y/o la microcirugía de laringe con láser de $\mathrm{CO}_{2} \mathrm{O}$ microinstrumental frío y posterior topicación con mitomicina-C.

Se definieron como éxito quirúrgico aquellos pacientes decanulados y como fracaso quirúrgico, cuando continuaron con el tubo de Montgomery (TM) o traqueostomía. ${ }^{3}$

\section{RESULTADOS \\ Datos demográficos}

Se recolectaron 187 pacientes con diagnóstico de ES, de los cuales se excluyeron 34 por no cumplir con los criterios de inclusión de tratamiento, 3 por fallecimiento (debido a patología oncológica) y 2 por falta de seguimiento. El número total fue de 148 pacientes, 67 (45,3\%) 
mujeres y 81 (54,7\%) varones, uno de ellos, un feto al que se le realizó un procedimiento EXIT (tratamiento exútero-intraparto) por presentar diagnóstico prenatal de atresia laríngea.

La media de edad fue 4,6 años; el rango etario más común fue entre el año y los 5 años de vida. La causa más frecuente de ES fue la adquirida, que se correspondió en un $86,5 \%$ a la intubación endotraqueal; el 0,7\% (1 paciente) presentó un síndrome inhalatorio; otro paciente, un tumor de laringe (tumor miofibroblástico inflamatorio subglótico); y otro, un traumatismo laríngeo. El 11,4\% (17/148) presentó ES congénita.

\section{Asistencia respiratoria mecánica prolongada}

Hubo 128 pacientes con antecedente de asistencia respiratoria mecánica prolongada (ARM), 63 (50\%) pacientes por enfermedad respiratoria baja, $13(10 \%)$ pacientes por cardiopatía congénita, $6(4,5 \%)$ pacientes por presentar quemaduras, $5(4 \%)$ pacientes por encontrarse en shock séptico, 5 (4\%) pacientes por sufrir de síndrome de aspiración de líquido amniótico meconial, 4 (3\%) pacientes por presentar tumor o malformación craneofacial, $3(2 \%)$ pacientes por laringomalacia, $3(2 \%)$ pacientes por posoperatorio (atresia esofágica, cirugía de Nissen, hernia diafragmática), 4 (3\%) pacientes por presentar síndrome de GuillainBarré, $3(2 \%)$ pacientes por traumatismo encéfalocraneano, $2(1,5 \%)$ pacientes por accidentes automovilísticos y $17(14 \%)$ pacientes por causas misceláneas. El tiempo de ARM fue de 3 a 180 días; media de 36,67 días.

\section{Cuadro clínico}

El estridor inspiratorio o bifásico fue el síntoma prevalente. Otros fueron disfonía o afonía, disnea, tiraje y uso de músculos respiratorios accesorios. Dichos síntomas acontecían en reposo o al realizar ejercicio físico.
Tipos de estenosis subglóticas (según el diagnóstico endoscópico)

La mayoría de las ES correspondieron a grado II $(30 \%)$ y grado III (31\%); 21 fueron estenosis grado I (12\%); 41, grado IV (22\%); y $10(5 \%)$ pacientes presentaban edema subglótico con fibrina y erosión del cartílago cricoideo.

\section{Tratamiento (procedimiento y/o cirugía)}

A 18 se los controló evolutivamente realizando observaciones clínica-endoscópicas periódicas.

A 56 de 148 (31\%) pacientes se les realizaron dilataciones. De estos, la mayoría presentaba ES grado II y III. A 9 pacientes se les realizó más de 1 dilatación, entre 2 a 6 dilataciones, media de 2,7. Los resultados obtenidos con esta terapéutica fueron mejores en las ES grado II.

Se operaron pacientes de 1 a 21 años, media de 6,2 años, con una distribución simétrica. 74 de 148 (40\%) pacientes fueron sometidos a cirugía laríngea a cielo abierto. Se realizaron $65(78 \%)$ cirugías de expansión laríngea con laringotraqueofisura y colocación de injerto cartilaginoso y tubo de Montgomery (TM); 7 laringotraqueofisuras y colocación de TM; 2 laringotraqueofisuras con injerto cartilaginoso y tubo nasotraqueal (TNT); 3 laringotraqueofisuras con injerto cartilaginoso y Rutter; una laringotraqueofisura con colocación de molde laríngeo y otra laringotraqueofisura con colocación de Rutter.

El TNT, el Rutter y el molde laríngeo se consideran prótesis laríngeas. Se realizaron tres resecciones cricotraqueales. Nueve de 74 pacientes requirieron más de una cirugía laríngea abierta, por lo tanto, la cantidad total fue de 83. En una paciente, se realizó colocación de homoinjerto de aorta, ya que la sutura de la anastomosis cricotraqueal sufrió una dehiscencia. Se utilizó, más frecuentemente, como injerto el costal anterior; en 61 cirugías, se empleó la parrilla costal

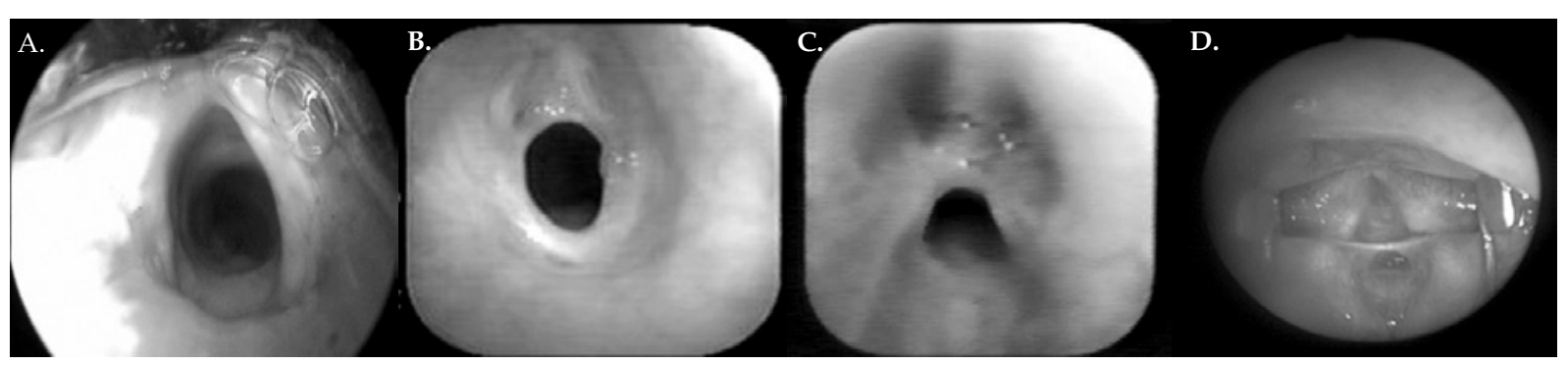


como zona donante y la laringotraqueofisura anterior como sitio receptor.

\section{Tiempo de permanencia de tubo de Montgomery /prótesis laríngea}

El TM permaneció colocado por un rango de 1 a 24 meses, media de 10,75 meses. El molde laríngeo, por 2 meses. El TNT, por un rango de 3 a 4 días, media de 3,5 días, y el Rutter, por un período de 1 a 4 meses, media de 2 meses.

\section{Complicaciones posquirúrgicas}

De 74 pacientes intervenidos, 32 manifestaron granulomas proximales y/o distales al TM, que requirieron su resolución endoscópica. Otras complicaciones menos frecuentes fueron cuadro aspirativo (por tener el TM en una posición más alta de lo indicado), infección de la herida cervical, neumonía, enfisema subcutáneo y neumotórax. Un paciente sufrió de la extrusión del injerto costal anterior y dehiscencia de la sutura cervical, que se resolvió quirúrgicamente. La complicación posquirúrgica más apremiante fue la dehiscencia de la sutura de una anastomosis cricotraqueal término-terminal, que requirió la colocación de homoinjerto de aorta porque los cabos remanentes eran insuficientes.

\section{DISCUSIÓN}

Las ES adquiridas son más frecuentes que las congénitas; en los últimos años, se ha registrado un incremento en su incidencia debido al uso de ARM. Esto, aunque sea por cortos períodos de un tubo endotraqueal (TET), la insuflación excesiva del balón que sobrepase la perfusión capilar, los episodios de hipotensión arterial y los tiempos prolongados de intubación sumados a características propias de la anatomía laríngea provocan isquemia, edema, necrosis y ulceración con granulación y cicatrización fibrosa. ${ }^{1-5}$ Por lo tanto, de ser posible, se debe utilizar un TET sin balón en niños menores de 3 años y, ante la necesidad de usar TET con balón, se sugiere el control diario de la presión en menos de $20 \mathrm{~cm} \mathrm{H}_{2} 0$ y posterior control radiológico del tubo para verificar que el balón se encuentre en la tráquea. Mantener una sedación adecuada evita el movimiento del niño y del tubo, y disminuye el riesgo de lesión.

Otras causas menos comunes de ES adquirida son reflujo gastrolaríngeo (RGL), infecciones, desórdenes inflamatorios y/o reumatológicos crónicos, traumas laríngeos externos, quemaduras térmicas y químicas, lesiones posoperatorias de cirugías laríngeas, tumores laríngeos y la realización de una traqueostomía alta (a nivel del cartílago cricoideo). ${ }^{1-7}$

Dentro de la valoración del niño con ES, es de suma importancia la realización de $\mathrm{pH}$ e impedanciometría para evaluar RGL por el impacto negativo de este sobre las estructuras laringotraqueales, ya que es causa de ES y de fracaso terapéutico. Por lo tanto, su tratamiento médico y hasta quirúrgico debe tenerse en cuenta. Algunos autores realizan la evaluación gastroenterológica si observan signos indirectos de RGL durante la valoración de la vía aérea. ${ }^{3}$

La conducta terapéutica irá desde la observación clínico-endoscópica en estenosis leves y en pacientes sin dificultad respiratoria ni trastornos en la deglución hasta las cirugías laríngeas a cielo abierto en las ES moderadas/graves. ${ }^{8}$

Las dilataciones se reservan para las ES sintomáticas grado I y grado II membranosas. La mitomicina $\mathrm{C}$ es un medicamento oncológico, inhibidor de los fibroblastos y de la cicatrización, que colabora en la resolución de estenosis agudas. ${ }^{4}$

En la literatura, se encuentran diversas técnicas quirúrgicas para la corrección de la ES, al igual que el uso de distintas prótesis laríngeas. ${ }^{5}$ Dichas cirugías comprenden la realización de laringotraqueofisura con o sin colocación de injerto y/o prótesis laríngea y hasta la resección cricotraqueal, publicada por Monnier, et al. en 1993. ${ }^{9}$ En general, la laringotraqueofisura con injerto costal es el tratamiento de elección para las ES grado II, III y IV de Cotton, con utilización de injerto anterior y posterior según la gravedad de la estenosis. ${ }^{10,11}$ La laringotraqueofisura es menos compleja y extensa que la resección cricotraqueal, ya que no requiere de movilización traqueal. ${ }^{5} \mathrm{La}$ resección cricotraqueal está indicada en las ES grado III graves y grado IV de Cotton y en los fracasos de cirugías expansivas.

El propósito de la prótesis laríngea es ayudar a mantener el área reconstruida y el injerto en posición. ${ }^{3}$ Hemos tenido buenos resultados con el tubo de Montgomery, que debió posicionarse en la comisura posterior, por encima de las cuerdas vocales (Figura 3).

El objetivo final del tratamiento de las ES es lograr una ventilación adecuada, mejorar las posibilidades de comunicación y la decanulación definitiva, de ser posible antes del ingreso escolar, lo que disminuye el riesgo de muerte al no perpetuar la traqueotomía en el niño con una lesión grave de la laringe.

Sobre la base de nuestra experiencia, se realizan las siguientes recomendaciones: 


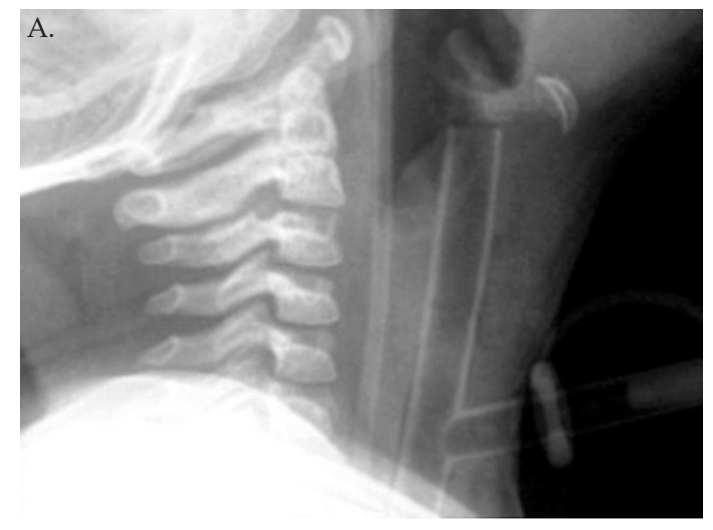

- Valorar la vía aérea en todo paciente con disfonía, dificultad respiratoria y/o fallos en la extubación.

- La valoración gastroenterológica y/o pH e impedanciometría es indispensable en pacientes con ES.

- Las dilataciones se deben reservar para las ES grado I y II de Cotton. Luego de tres dilataciones fallidas, se aconseja realizar una traqueostomía para asegurar la vía aérea del paciente.

- Las cirugías expansivas han demostrado ser la intervención de elección en las ES, y, en caso de que estas fallen, se deben tener en cuenta las cirugías de resección.

\section{CONCLUSIONES}

De 148 pacientes incluidos en el trabajo, el 86,5\% presentó como causa de ES trauma por intubación endotraqueal.

Los 18 pacientes controlados evolutivamente presentaron mejoría sintomática, gracias al progreso pondoestatural y/o al uso de corticoides inhalados y/o tratamiento antirreflujo.

De 56 pacientes tratados con dilatación, 38 $(67,8 \%)$ lograron su curación.

De 74 pacientes sometidos a cirugía laríngea a cielo abierto, 40 (54\%) se encontraban decanulados al finalizar el estudio. A otros 27 (37\%) pacientes aún no se les retiró el TM por incumplimiento en el tiempo posoperatorio. Un niño con síndrome de Down permanece con TM por glosoptosis. Seis pacientes continúan traqueostomizados por fracaso quirúrgico: la paciente portadora del homoinjerto aórtico, 4 niños por RGL y 1 paciente por síndrome aspirativo. Las complicaciones posquirúrgicas más frecuentes son los granulomas

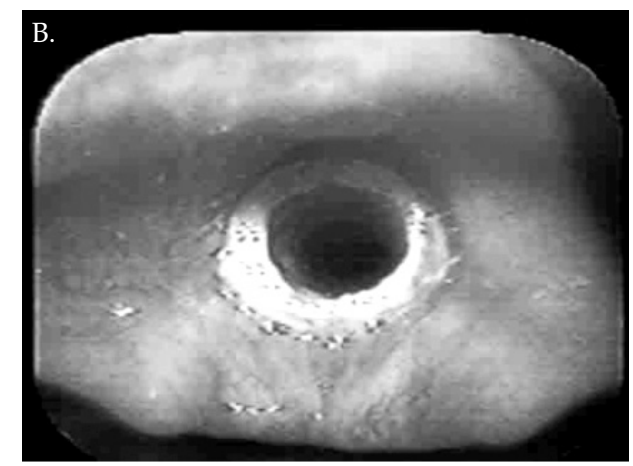

peri-TM, que pueden obstruir el TM y provocar dificultad respiratoria. Es de suma importancia la radiografía de cuello para su diagnóstico y la endoscopía para su resolución.

\section{REFERENCIAS}

1. Lusk R, Wolley A, Holinger L. Laryngotracheal stenosis. En Holinger LD, Lusk RP, Green CG, eds. Pediatric Laryngology and Bronchoesophagology. Philadelphia: LippincottRaven;1997:165-86.

2. Willging JP, Cotton RT. Subglottic stenosis in the pediatric patient. En Myer CM, Cotton RT, Shott SR, eds. The Pediatric Airway: an Interdisciplinary Approach. Philadelphia: Lippincott;1995:111-32.

3. Preciado D. A randomized study of suprastomal stents in laryngotracheoplasty surgery for grade III subglottic stenosis in children. Laryngoscope 2014;124(1):207-13.

4. Choo KKM, Tan HKK, Balakrishnan A. Subglottic stenosis in infants and children. Singapore Med J 2010;51(11):848-52.

5. Bajaj Y, Cochrane LA, Jephson CG, Wyatt ME, et al. Laryngotracheal reconstruction and cricotracheal resection in children: recent experience at Great Ormond Street Hospital. Int J Pediatr Otorhinolaryngol 2012;76(4):507-11.

6. Monnier P. Acquired post-intubation and tracheostomyrelated stenoses. En Monnier P, ed. Pediatric airway surgery: management of laryngotracheal stenosis in infants and children. Berlin: Springer;2011:183-98.

7. Zanetta A, Tiscornia C, Rodríguez H, Cuestas G. Estenosis subglótica: Reconstrucción laringotraqueal con injerto de cartílago posterior en niños. FASO 2010;17(1):33-8.

8. Okamoto M, Nishijima E, Yokoi A, Nakao M, etal. Strategy for surgical treatment of congenital subglottic stenosis in children. Pediatr Surg Int 2012;28(11):1115-8.

9. Monnier P, Savary M, Chapuis G. Partial cricoid resection with primary tracheal anastomosis for subglottic stenosis in infants and children. Laryngoscope 1993;103(11 Pt1):127383.

10. Zanetta A, Rodríguez H, Botto H, Nieto M, et al. Estenosis laríngeas en pediatría. Reconstrucción laringotraqueal como tratamiento. Anales Otorrinolaringológicos del Perú 2012;17:77-87.

11. Zanetta A, Tiscornia C, Rodríguez $\mathrm{H}$, Botto $\mathrm{H}$, et al. Resolución quirúrgica de las estenosis laringotraqueales en pediatría. FASO 2011;18(2):64-71. 